\title{
2.2. Medien und Medienkommunikation
}

\author{
Axel Schmidt
}

Keywords: Kontakt, Medienbegriff, Medienspezifik, Mediensystematik, Mittelbarkeit

\section{Einleitung}

Der vorliegende Beitrag erörtert den Begriff der Medien aus mediensoziologischer Perspektive. Soziologie befasst sich mit dem Zustandekommen von sozialer Ordnung durch Prozesse der Interaktion und Kommunikation (s. Schmidt in diesem Band), Mediensoziologie mit der Rolle ,der Medien` innerhalb solcher Prozesse (vgl. Jäckel, 2005 a). Grundsätzlich vermitteln Medien soziale Austauschprozesse und machen sie dadurch wahrnehmbar, was Soziales für wissenschaftliche Beobachter überhaupt erst zugänglich macht. Da daher in einem weiten Verständnis jeder Interaktions- und Kommunikationsprozess auf Medien angewiesen ist, ergibt sich die Notwendigkeit einer Engfübrung des Medienbegriffs. Soll Mediensoziologie nicht in einer Untersuchung sozialer Austauschprozesse und damit im Gegenstandsbereich der Soziologie im Allgemeinen aufgehen, müssen mediensoziologische Fragestellungen darauf fokussieren, wie sich Prozesse der Interaktion/Kommunikation verändern, wenn sie in einem technischen Sinn medial vermittelt werden. Dabei vermitteln Medien nicht bloß, sondern prägen und verändern soziale Austauschprozesse tief greifend (McLuhan, 1970). Dies ist insbesondere auf eine medienspezifische Variation grundlegender Rahmenbedingungen kommunikativen/interaktiven Austauschs zurückzuführen: Der Einsatz von Medien verändert etwa Zeit- und Raumverhältnisse, wechselseitige Wahrnehmbarkeit und Zugänglichkeit sowie Zeichenverwendung und Kommunikationszwecke. Die Anfänge einer Mediensoziologie hängen daher mit dem Entstehen und der zunehmenden Verbreitung medial und insbesondere massenmedial vermittelter Kommunikation zusammen (Jäckel, 2005 b), welche sich aber erst auf dem Hintergrund eines - dann wiederum aus der Blickrichtung ,der Medien' - als unvermittelt konzipierten Kontakts profilierten.

Interaktion und Kommunikation sind als Austauschprozesse auf Kontakt angewiesen oder - wie Graumann (1972, S. 1117) es gefasst hat - setzen voraus, dass „Menschen voneinander Kenntnis nehmen “. Konkrete Austauschprozesse sind daher immer in irgendeiner Weise situiert, d.h., sie finden unter bestimmten raum-zeitlichen (Situation; Unmittelbarkeit/Mittelbarkeit), dinglich-körperlichen (Kontaktmodalitäten; Artefakte/Speichermedien) und medial-kommunikativen (Kommunikationsmittel/Zeichentypen; Art des Kanals) Bedingungen statt. Dabei hängt es von der je konkreten Situierung des Austauschprozesses ab, welche Kontaktmodalitäten und Kommunikationsmittel bzw. -kanäle möglich sind bzw. Verwendung finden können. Insofern entsteht der Begriff der Medien an der Schnittstelle von Interaktion/Kommunikation (Austausch) und Situation (Raum/Zeit-Verhältnisse) und hebt ab auf die grundsätzliche 
Einsicht, „dass jegliche (menschliche/soziale) Kommunikation eines Mittels bedarf, durch das hindurch oder mithilfe dessen kommuniziert wird. [...]. Medien sind also zunächst und grundsätzlich immer auch ,Elemente von Kommunikation', sie treten zwischen die Menschen und trennen sie gewissermaßen, indem sie sie verbinden" (Mock, 2006, S. 188).

Aus mediensoziologischer Perspektive wird der Medienbegriff also erst in dem Moment virulent, in dem soziale Austauschprozesse das Hier und Jetzt beständig und massenhaft zu übersteigen vermögen und verweist damit auf die Genese der sogenannten Massenmedien (Presse, Kino, Radio, Fernsehen) bzw. der massenhaft genutzten Individualmedien zur Unterstützung technisch vermittelter interpersonaler Kommunikation (Telefon/Handy), welche heute in den sogenannten Interaktionsmedien (Computer/Internet/Web 2.0) konvergieren. Erst von der Warte einer massenhaft medial geprägten Kommunikation aus profilierte sich die Medienspezifik von Faceto-Face-Interaktionen als unmittelbar, kopräsent, flüchtig, simultan, mehrkanalig, multimodal etc. überhaupt erst und diente so gegenläufig als Folie der Bestimmung medialer Kommunikationsformen ${ }^{1}$.

Wie viele analytische Grundbegriffe lässt sich auch der Medienbegriff nicht eindeutig festlegen, da eine Reihe von Disziplinen ihn in sehr unterschiedlicher Weise gebrauchen. ${ }^{2}$ Zudem - so Ziemann (2006) - "[gibt es] eine Basisdefinition noch nicht" (ebd., S. 16), sodass die meisten Beiträge, die sich in systematischer Weise mit dem Begriff der Medien befassen, Verwendungsweisen des Medienbegriffs bestimmen. Diese Diskussion soll im Folgenden skizziert werden (2), um im Anschluss daran Medien hinsichtlich ihrer Funktionen zu systematisieren (3) und - darauf aufbauend gesellschaftliche Leistungen zu skizzieren (4).

\section{Verwendungsweisen des Medienbegriffs}

Einen Ansatzpunkt für die Begriffsbestimmung liefert ein Blick auf die Bedeutung und Wortherkunft: „Medium/Medien“ (lateinisch: medium, die Mitte, das Mittlere) bedeutet Mittel, Mittelglied oder Mittler(in), vermittelndes Element (vgl. hierzu Mock, 2006, S. 185ff.). Mittel verweist auf den Gebrauchs- bzw. Werkzeugcharakter (A benutzt X um Y zu erreichen). Mittelglied auf die dadurch eingenommene struktu-

1 Schütz/Luckmann (1979, S. 92) geben zu bedenken: „Obwohl wir von der ,unmittelbaren` Erfahrung des Mitmenschen sprechen, ist natürlich auch diese Erfahrung im strengsten Sinne des Wortes ,vermittelt'. Den Erlebnisablauf des Mitmenschen erfasse ich nur ,mittelbar', indem ich seine Bewegungen, seinen Ausdruck, seine Mitteilungen als Anzeichen von subjektiv sinnvollen Erfahrungen eines fremden Ichs auslege“. Dennoch: "Unter all meinen Erfahrungen eines fremden Ichs ist aber die Begegnung des Mitmenschen in der Gleichzeitigkeit der Wir-Beziehung die gleichsam am Wenigsten mediatisierte" (ebd.).

2 So plädieren kulturwissenschaftliche und insbesondere medienphilosophische Ansätze etwa für eine Öfnung des Begriffs weit über den Geltungsbereich als (technisches) Kommunikationsmittel hinaus (vgl. hierzu etwa Tholen, 2005 sowie Münker \& Rösler, 2008), während innerhalb sozialwissenschaftlicher Ansätze (vgl. für viele etwa Faulstich, 2004) eine Einschränkung angestrebt wird (etwa auf technische Kommunikationsmittel). 
relle Position zwischen zwei anderen Elementen (A - X - B). Da Medien aber mehr sind als Mittel oder Werkzeuge zu Erreichung bestimmter Zwecke, mithin also als spezifische Mittel zu begreifen sind (vgl. hierzu u.a. Böhme-Dürr, 2003, S. 358; Habscheid, 2000, S. 127; Hickethier, 2003, S. 19), ergibt sich der entscheidende Bedeutungsaspekt aus der Komponente "vermittelndes Element", welche auf die Funktion der Vermittlung abhebt. Das heißt, zwischen ein $\mathrm{Zu}$-Vermittelndes (etwa ein Gegenstand der äußeren Welt) und ein Vermitteltes (etwa der wahrgenommene Gegenstand) tritt ein Vermittelndes (etwa die menschlichen Sinne und die kognitive Verarbeitung von Wahrgenommenem), welches den Prozess der Vermittlung ermöglicht und prägt. Der Begriff der Vermittlung hebt dabei im Gegensatz zum Mittel- oder Werkzeug-Begriff weniger auf die Zielerreichung (Mittel zum Zweck) oder die Formung eines Ursprungszustandes (Werkzeug) ab, als vielmehr auf die Ermöglichung und Katalyse von Prozessen der Welterzeugung (Wahrnehmung, Kommunikation, Kognition). ,Medium/Medien' werden daher - wie eingangs erwähnt - in einem allgemeinen Sinn häufig als Mittel der Wahrnehmung, Entäußerung und Kommunikation gefasst. So etwa Hickethier (2010, S. 19): „Entscheidend ist also, dass es sich bei den Medien um Mittel handelt zur Formulierung von Gedanken, Gefühlen, Inhalten sowie von Erfahrung über die Welt". Unterstellt man, dass dies zeichenhaft geschieht, „[bezieht sich] der Begriff ,Medium“ auf Kommunikationsmittel, also auf die Mittel zur Weitergabe von Zeichen" (Böhme-Dürr, 2003, S. 358). Medien sind dann nicht zwingend Mittel solcher Prozesse (im Sinne von Instrumenten) (vgl. Habscheid, 2000, S. 127), sondern eher Vermittler, das heißt sie ermöglichen und prägen die vermittelten Prozesse in einem sehr weitgehenden Sinn ${ }^{3}$. Eine solche, auf die Prägekraft von Vermittlungsleistungen abhebende Fassung des Medienbegriffs schlägt etwa Posner (1985, S. 255) vor, wenn er Medium als ein „System von Mitteln für die Produktion, Distribution und Rezeption von Zeichen, das den in ihm erzeugten Zeichenprozessen bestimmte gleichbleibende Beschränkungen auferlegt" definiert. Beides - Medium im Sinne von Mittel und im Sinne von Vermittler - macht deutlich, „dass es sich bei ,Medium' nicht um einen Substanzbegriff mit einem eindeutigen Bezug zu einem ,Realobjekt' handelt, sondern vielmehr um einen Beziehungs- oder Funktionsbegriff (Mock, 2006, S. 184). Daher gilt: „ein Medium in diesem Sinne ist immer nur ,Medium' in Bezug auf etwas anderes, innerhalb eines bestimmten kommunikativen Kontextes" (ebd., S. 189). Verwendungsweisen des Medienbegriffs ergeben sich daher aus der Vorstellung einer Vermittlungsrelation mit spezifischen Kapazitäten (Möglichkeiten und Beschränkungen), womit gängige Bezeichnungen medialer Kommunikationstypen korrespondieren. Folgende Verwendungsweisen lassen sich in Anlehnung vor allem an Posner (1985) und Mock (2006) unterscheiden ${ }^{4}$ :

3 So besteht ein wesentlicher Grundzug des Medienbegriffs darin - je nach Weite der Verwendung oder Sphäre, auf die er bezogen wird (Wahrnehmung, Zeichen, Kommunikation, Technik etc.) -, die Abhängigkeit von Materialitäten einzuklagen, denen Prozesse der Bedeutungsgenerierung aufgrund ihrer Medienvermittlung unweigerlich unterworfen sind (vgl. grundsätzlich Gumbrecht \& Pfeifer, 1988). Siehe auch unten die Erläuterungen zum Konzept „Mediendispositiv“.

4 Zugegriffen wurde des Weiteren auf Darstellungen bei Böhme-Dürr (2003), Habscheid (2000), Hickethier (2010), Kübler (2000), Leschke (2007), Sandbothe (2003) Schmidt (1994), Tholen (2005) und Ziemann (2006). 


\subsection{Naturwissenschaftlicher Medienbegriff}

\section{Medien als Mittel der Wahrnehmung}

Wahrnehmung kann als Voraussetzung für Kommunikation gelten und setzt ein wahrnehmendes Subjekt sowie ein wahrnehmbares Objekt voraus; entsprechend lassen sich ein biologischer und physikalischer Medienbegriff unterscheiden ${ }^{5}$ :

2.1.1 Biologischer Medienbegriff: bezieht sich auf die Körperorgane, die am Vermittlungsprozess beteiligt sind (Rezeptionsorgane/Sinnesmodalitäten; Produktionsorgane);

- Beispiel: Auge bzw. Seh-Apparat oder Artikulations-Apparat (Sprachproduktion);

- Medialer Kommunikationstypus: etwa auditive und visuelle Medien; dabei ist der Rezeptionskanal ausschlaggebend (vgl. Mock, 2006, S. 189 f.) ${ }^{7}$.

2.1.2 Physikalischer (chemischer) Medienbegriff: bezieht sich auf die chemischen Elemente und deren physikalischen Zustände, die am Vermittlungsprozess beteiligt sind und einen Kontakt zwischen kommunizierenden Instanzen ermöglichen (Träger- bzw. Kontaktmaterie);

- Beispiel: die Luft als Trägermedium bzw. Schallwellen als Kontaktmaterie der Lautübertragung (im Falle auditiver Sinneswahrnehmung); elektromagnetische Felder als Trägermedium bzw. elektromagnetische Wellen (Licht) als Kontaktmaterie (im Falle visueller Sinneswahrnehmung);

- Medialer Kommunikationstypus: etwa optische oder akustische Medien.

Biologischer (Wahrnehmung/Sinnesorgane) und physikalischer (Kontakt-/Trägermaterie) Medienbegriff lassen sich nun im Sinne eines Kanals verkoppeln, sodass den fünf menschlichen Hauptsinnen entsprechend optisch-visueller, akustisch-auditiver,

5 Beide Begriffe bezeichnen entweder gängige mediale Kommunikationstypen, etwa visuell (biologisch) oder optisch (physikalisch) (vgl. Posner, 1985) oder aber Realobjekte, etwa Auge/Ohr (biologisch) oder elektromagnetische Wellen/Felder bzw. Luft/Schallwellen (physikalisch).

6 Als sinnliche Wahrnehmungsmedien lassen sich die Anschauungsformen von Raum und Zeit als auch die menschlichen Sinnesorgane begreifen (vgl. Sandbothe, 2003). Die Artikulationsorgane und der Körper als (An-)Zeichenproduzent sind zwar als biologisch einzustufen und spielen als Signalproduzenten eine Rolle, gehören jedoch - folgt man der Klassifikation von Sandbothe (2003) - eher zu den Verbreitungs- als zu den Wahrnehmungsmedien, da sie der Produktion nicht der Rezeption von Informationen/Zeichen dienen.

7 Siehe hierzu insbesondere auch Wulff (1978), der in seiner Begriffsbestimmung von Kanal den Weg eines Signals von einem Sender zu einem Empfänger zugrunde legt und unterscheidet zwischen Signal-Produktion, Kanal-Eingang, Kanal, Kontaktmaterie/Feld, Kanal-Ausgang und Signal-Rezeption. Setzt man dabei am Ende des Prozesses, also bei der Rezeption von Signalen an, lassen sich Signale danach unterscheiden, welche Sinnesmodalitäten sie ansprechen (Licht/ Auge=visuell, Schall/Ohr=auditiv etc.). Solche Differenzierungen sind für den Produktionsprozess weitaus problematischer, sodass die Differenzierung medialer Kommunikationstypen mit Blick auf die biologische Ausstattung des Menschen eher am Rezeptions- als am Produktionsprozess ansetzt. Unter der Perspektive des ,Kanals' hängt beides im Sinne eines Übermittlungsprozesses von Signalen natürlich zusammen (vgl. die Übersicht in Wulff, 1978, Abb. 6).

8 Bisweilen werden die Kontaktmaterie (Schall/Licht) und deren Trägermedien (Luft/elektromagnetische Felder) (vgl. Posner, 1985), bisweilen nur Letzteres (vgl. Böhme-Dürr, 2003) zum Bezugspunkt eines physikalischen Medienbegriffs. 
chemisch-olfaktorischer, chemisch-gustatorischer sowie mechanisch-taktiler Kanal unterschieden werden können'. Ausgehend von einer solchen Zuordnung menschlicher Sinne und physikalischer Reizqualitäten im Sinne von Wahrnehmungs- oder Kommunikationskanälen lassen sich Zeichen(Systeme) und technische Medien hinsichtlich ihrer Kanalkapazität etwa als optisch-visuelle (z.B. Schrift) oder als audiovisuelle Medien (etwa Fernsehen) fassen. Die Beschaffenheit der gegenständlichen Welt und ihre Wahrnehmung ist die Grundlage menschlicher Existenz. Kübler (2000) spricht daher von einem „universalen Medienbegriff“ (ebd., S. 6).

\subsection{Sozialwissenschaftliche Medienbegriffe}

\subsubsection{Medien als Mittel der Kommunikation oder semiotisch-kommunikativer bzw.} kodebezogener Medienbegriff

Dieser bezieht sich auf Zeichen(-systeme) oder Kodes, mithilfe derer Bedeutung konstruiert bzw. transferiert wird und die Voraussetzung dafür sind, dass Mentales (Gedanken, Bedeutungen) in materiell wahrnehmbare Signale umgesetzt werden kann. Einem solchen „elementaren Medienbegriff“ (Kübler, 2000, S. 6) zufolge „sind alle Entäußerungen oder Manifestationen des Geistes medial, weil sie mittels eines Zeichensystems artikuliert und damit materialisiert werden" (ebd.). Dabei wurzelt der Zeichenträger oder Zeichenkörper (vgl. Böhme-Dürr, 2003, S. 364 f.) in der materiellen Welt, hat also - wie Mock (2006, S. 191) betont - „eine materiell-energetische Dimension (Signalstruktur)“, weshalb Zeichen(systeme) „auf den zuvor beschriebenen physikalischen Medien auf[setzen]" (ebd.) ${ }^{10}$.

\subsubsection{Technologischer Medienbegriff oder Medien als Mittel der Distribution/Verbreitung von Informationen/Zeichen}

Informationen/Zeichen können mithilfe technischer Mittel erzeugt, weitergegeben und/oder fixiert werden, die - abgesehen von materiellen Medien (etwa Höhlenwände oder Sand) - als „technische Artefakte“ (Mock, 2006, S. 191) begriffen werden können. Technik ist dabei „als eine Einheit von Apparaten, Anlagen, Geräten und ähnlichem, kurz: als Einheit zweckhafter Artefakte“ (Böhme-Dürr, 2003, S. 362) zu verstehen. Bezogen auf Kommunikation bzw. Zeichenprozesse sind hierunter jene technischen Mittel zu verstehen, „die bei der Erzeugung von Zeichenprozessen zur

9 Vgl. hierzu Böhme-Dürr (2003, S. 363 f.), Holly (1996, 1997) und Mock (2006, S. 189 f.). Unter Kanal wird der Transportweg der Signale verstanden. Dabei werden als Kanal bisweilen die menschlichen Sinne (etwa auditiver Kanal) oder Artikulationsorgane (etwa vokaler Kanal), die Kontaktmaterie bzw. die physikalische Qualität der Signale (etwa akustischer Kanal, z.B. Schallwellen), der materielle Träger als solcher (etwa Luft für Schallwellen) oder deren Kombination verstanden. Böhme-Dürr (2003) etwa spricht vom „vokal-auditiven“ bzw. „artikulatorisch-auditiven" Kanal (ebd., S. 364). In einem engeren Verständnis bezieht sich Kanal auf „dasjenige materielle Medium, das Signale vom Sender zum Empfänger übertragen kann“ (vgl. Wulff, 1978, S. 37), also auf Trägermedien wie gasförmige, flüssige oder feste Stoffe (etwa Luft), sodass die Begriffe ,Kanal' und ,Medium' entsprechend einer physikalischen Auffassung des Medienbegriffs in eins fielen.

$10 \mathrm{Vgl}$. hierzu grundlegend die Unterscheidung von Signifikant (Zeichenträger) und Signifikat (Zeichenbedeutung) bei Ferdinand de Saussure (1967, S. 76 ff.). 
Modifikation der Kontaktmaterie eingesetzt werden, die die physische Verbindung zwischen dem Produktionsorgan des Senders und dem Rezeptionsorgan des Empfängers herstellt“" (Posner, 1985, S. 362). Je nach Umfänglichkeit des Technikeinsatzes lassen sich primäre (kein Technikeinsatz), sekundäre (Technikeinsatz auf Kommunikatorenseite) und tertiäre (Technikeinsatz auf Kommunikatoren- und Rezipientenseite) Medien unterscheiden ${ }^{11}$. Je nach technischer Komplexität sind Medien technische Hilfsmittel der Erzeugung, Speicherung, Übertragung und Verarbeitung von Informationen/Zeichen.

\subsubsection{Medien als systemischer Zusammenhang}

Aspekte der Wahrnehmung und Beschaffenheit der gegenständlichen Welt sowie Zeichensysteme und mediale Techniken "stellen in [ablaufenden Kommunikationen] [...] einen untrennbaren, systemischen Zusammenhang dar, in dem die Mittel der einen Ebene ,Voraussetzung ' für die Elemente der anderen Ebenen sind bzw. diese konstituieren“ (Mock, 2006, S. 193). Auf diese Weise kommt es zur Institutionalisierung von Einrichtungen medialer Kommunikation sowie von kommunikativen Formen (Gattungen, Genres) ${ }^{12}$. „,Medium ' in diesem vierten Sinne meint also, dass der Zusammenhang von Kommunikationsmitteln, Akteuren, Themen, darauf bezogenen Regelungen etc. als etablierte, sozial anerkannte Art und Weise zu kommunizieren, zum eigenwertigen Zusammenhang, zum ,Medium', wird“ (ebd., S. 193 f.). Hieran knüpfen sich zwei weitere Medienbegriffe:

- Soziologischer Medienbegriff: bezieht sich auf die „sozialen Institutionen, die die biologischen, physischen und technischen Mittel mit dem Ziel der Erzeugung von Zeichenprozessen organisieren" (Posner, 1985, S. 256). Dies kann sehr weit ausgelegt werden, sodass dies - wiederum je nach Extension des Kommunikationsbegriffs - alle sozial geregelten Formen der Affizierung menschlicher Sinne umfasst. Im engen Sinne sind hiermit allerdings Organisationen zur Herstellung und Verbreitung von Kommunikationsangeboten gemeint (etwa das Medium ,Fernsehen').

- Kulturbezogener Medienbegriff: bezieht sich auf den „Zweck der Botschaften“ (Posner, 1985, S. 256) und verweist damit auf Konzepte wie (kommunikative) Gat-

11 Vgl. grundlegend Pross (1972). Die Kategorie der primären oder „Menschmedien“ (vgl. Faulstich, 2004, S. 23 ff.) ist streng genommen kein Element des technischen Medienbegriffs (vgl. Böhme-Dürr, 2003, S. 363), wird allerdings in der Regel unter dem Technikaspekt von Medien mit aufgeführt. Neben einer Abgrenzungsfunktion (,Technik/keine Technik') verweist dies zugleich auf mediale Institutionen, bei denen eine Abgrenzung schwerfällt, wie etwa das Theater, wo trotz Kopräsenz Techniken zum Einsatz gelangen (etwa Bühne und Licht). Mock (2006) argumentiert, dass sich solche „Medien im Sinne von Medium als Form von Kommunikation" (ebd., S. 194) beschreiben lassen. Insgesamt verweist dies auf eine fließendere Konzipierung der Übergänge zwischen mediatisierten und nicht-mediatisierten Kommunikationsformen (siehe hierzu Schultz, 2001). Hierauf verweist etwa auch Auslander (2008) in seiner Auseinandersetzung mit dem Begriff "Liveness“.

12 Faulstich (2004, S. 12) spricht etwa von „einem institutionalisierten System um einen organisierten Kommunikationskanal von spezifischem Leitungsvermögen mit gesellschaftlicher Dominanz". Gattungen/Genres können als Orientierungssystem für Medienangebote gelten (vgl. hierzu grundlegend Schmidt (1994, S. 176 ff.) sowie Schweinitz, 1994). 
tung, Genre oder Textsorte (etwa Medium ,Roman'). „Der Kommunikationszweck“ - so Posner (1985) - ,verleiht den Botschaften unabhängig davon, durch welche biologischen, physischen, technischen und sozialen Medien sie laufen, gleichartige Strukturen“ (ebd., S. 256).

Auf der Grundlage der Differenzierung biologischer, physikalischer, semiotischer, technologischer, institutioneller und gattungstypologischer Zugänge zum Medienbegriff lassen sich weite und enge Fassungen unterscheiden, deren Pole sich wie folgt bestimmen lassen:

- weit: aiesthetische Auffassung von Medien, der zufolge Medien menschliche Wahrnehmung und damit auch Erkenntnis bedingen;

- eng: Medien als „dominante Kanäle“; der Fokus liegt auf technischer Vermittlung und gesellschaftlicher Relevanz (das betrifft insbesondere die sogenannten Massenmedien).

Eine für mediensoziologische Belange brauchbare Fassung des Medienbegriffs besteht darin, dass ausgehend von einem Begriffskern, nämlich Medien als technische Kommunikationsmittel sowie deren Produkte und Organisationsformen, weitere mediale Dimensionen miteinbezogen werden können ${ }^{13}$. Insofern hängt die evolutionäre Entstehung einer (neuen) technischen Kommunikationsform (wie etwa die der Fotografie, des Fernsehens oder des Internets) mit Prozessen der Manipulation von Wahrnehmbarem und darauf bezogener Wahrnehmung (Kanal) sowie mit Prozessen der Erzeugung und Verwendung von Informationen und Zeichen (Kode) zusammen. Der spezifische Zusammenhang von Kanal-, Kode- und Technikaspekten im Rahmen sozial institutionalisierter Kommunikationsformen übernimmt dabei - so Ziemann (2006, S. 17) - „eine besondere Problemlösefunktion“. Ein mediensoziologischer Begriff von Medien wäre daher an der Spezifik von durch technische Hilfsmittel hergestellter Kommunikation anzusetzen, wobei sich die spezifische Problemlösung vor dem Hintergrund der Abweichung von medienunvermittelter Kommunikation ergibt. Innerhalb eines solchen Funktionszusammenhangs lassen sich dann wiederum Querverbindungen zur ,Peripherie' des Medienbegriffs herstellen ${ }^{14}$. Ein solcher, für mediensoziologische Belange fokussierter Medienbegriff setzt am Konzept apparativ-technischer Vermittlung an und markiert Grenzen, insbesondere gegenüber

- Unmittelbarkeit/nicht technisch vermittelter Kommunikation (Face-to-Face-Kommunikation bzw. sogenannte Primär- oder Menschmedien);

- biologischen/physikalischen Voraussetzungen von Wahrnehmung/Kommunikation (,Kanal“);

13 Dies entspricht der Fassung des Medienbegriffs bei Schmidt (1994, S. 83ff.), allerdings unter Aussparung der Kommunikationsmittel/Zeichensysteme (etwa Sprache) als Medium.

14 Vgl. hierzu etwa Meyrowitz (1987), der seine gesellschaftlichen Diagnosen („Fernsehgesellschaft"; engl.: „no sense of place") an der Frage veränderter Wahrnehmungs- und Kommunikationsbedingungen durch technische Medienvermittlung ansetzt und auf dieser Grundlage zu weitreichenden Thesen gesellschaftlichen Wandels gelangt. 
- Verständigungsmitteln i.A. (Zeichen, Symbole, Sprache; häufig als Verständigungsoder Kommunikationsmittel bezeichnet) ${ }^{15}$;

- symbolisch generalisierten Medien in strukturfunktionalen bzw. systemtheoretischen Theorienbildungen ${ }^{16}$.

Ausgehend von einem solchen fokussierten Medienbegriff lassen sich unterschiedliche Medien hinsichtlich ihrer Funktionsweisen bestimmen.

\section{Funktionale Mediensystematiken}

\subsection{Grundfunktionen}

Nach Hiebel et al. (1998) lassen sich folgende medienlogische Grundfunktionen unterscheiden: Speicherung, Übertragung und Bearbeitung, die zu sechs Aspekten medialer Möglichkeiten erweitert werden können: Aufnahme, Speicherung, Übertragung, Vervielfachung und Reproduktion, Wiedergabe sowie Ver- bzw. Bearbeitung. Demzufolge lassen sich Aufnahme- bzw. Eingabemedien (etwa Kamera oder Stift), Speichermedien (etwa Filmstreifen oder Papier), Übertragungs- bzw. Transportmedien (etwa Fernsehen oder Post), Reproduktionsmedien (etwa fotografische Kopien oder Druck), Wiedergabemedien (etwa Diaprojektor) sowie Datenverarbeitungs- und Datengenerierungsmedien (etwa Computer) unterscheiden.

\section{2. "Rohdaten" und Kanalkapazität}

$\mathrm{Da}$ Medien als technische Hilfsmittel der Erzeugung und/oder Übermittlung von Zeichen begriffen werden können, ist ein weiteres Grundmoment medialer Funktionalität ihre Kanalkapazität. Damit ist das Vermögen von Medien gemeint, mit einem bestimmten Spektrum von Zeichen operieren zu können und damit bestimmte Kanäle anzusprechen. Dabei können Medien an unterschiedlichen ,Rohdaten ansetzen, und zwar prinzipiell entweder an primär digitalen/sprachlichen oder an analogen/sprachunabhängigen Informationen/Zeichen ${ }^{17}$. So können entweder uncodierte, analoge Daten (etwa Körperbewegungen im Stummfilm) oder bereits codierte Daten (etwa briefliche Kommunikation oder Printmedien, die Sprache verwenden) oder Kombinationen (etwa Gespräche im Fernsehen) medial übermittelt werden. Die mediale Auf-

15 Vgl. hierzu Habscheid (2000), der unter Rekurs auf Holly $(1996,1997)$ dafür plädiert, Zeichensysteme (etwa die Schrift) nicht als Medien zu fassen.

16 Ein solcher, auf die Annahmewahrscheinlichkeiten von Kommunikation zielender Medienbegriff, der etwa Geld, Liebe oder Macht als Medien begreift (vgl. zusammenfassend Schneider, 2002, S. 317 ff.), erscheint zwar theoretisch innovativ, ist jedoch zur Abgrenzung eines bearbeitbaren Gegenstandsbereichs wenig hilfreich (vgl. Leschke, 2007, S. 17 f.) und hat daher seine Relevanz eher in Bezug auf die Architektonik spezifischer Theorieentwürfe.

17 Mit „sprachlich/digital“ beziehen sich Hiebel et al. (1998) auf arbiträre Sprachzeichen, die sie als willkürlich gesetzt, diskret und konventionell begreifen und unterscheiden hinsichtlich des Kanals akustische/phonetische (etwa stimmliche Sprachlaute) und optische/visuelle (etwa Handschrift) Zeichen. „Optische und akustische Informationen analoger Natur" (Hiebel et al., 1998, S. 15) dagegen beruhen „auf proportionalen Wiedergabeverhältnissen oder Spiegelrelationen“ (ebd., 14), etwa filmbildliche Darstellungen von Körperbewegungen. 
zeichnung bzw. Übermittlung kann nun ihrerseits sowohl analog (d.h., die Daten werden ohne Zwischenschaltung einer Codierung aufgezeichnet) als auch digital (im Sinne eines Binärcode bzw. sekundärer Digitalität) erfolgen ${ }^{18}$. Obwohl digitale Verarbeitung analogen Daten ihr Spezifikum einer nicht-codierten Analogität nimmt, „bleibt doch Tönen und Bildern ihr wesentliches Charakteristikum - das der primär codefreien, analogen Wiedergabe der Daten des "Realen' - weitgehend erhalten“ (ebd., S. 27). Unterschieden werden können demnach analoge und digitale Medien mit Blick auf die zugrundeliegende Technik und analoge und digitale Darstellungsformen mit Blick auf die Informationsstruktur des Kommunikats ${ }^{19}$. Sprachliche Daten können in vokaler oder schriftlicher Form, analoge/sprachunabhängige Daten können als Laute/Geräusche oder als uncodiert Visuelles vorliegen. Während Schriftdaten bereits einer medialen Erzeugung und Speicherung unterliegen (etwa mittels Stift und Papier), sind vokale bzw. sprachunabhängige Daten zunächst flüchtig und medienunvermittelt ${ }^{20}$. Medien, die solche Informationen übertragen, verwenden entweder einen optischen (Schrift, Visuelles=optische Medien) oder einen akustischen (Laute/ Geräusche, gesprochene Sprache=akustische Medien) Kanal oder kombinieren beide (=audiovisuelle Medien). Die Übertragung kann im Falle der Schrift materiell (Transport: Brief/Post) oder elektronisch (etwa Telegrafie) erfolgen; Letzteres erfordert innerhalb der Medienkette eine Übersetzung in elektrische Signale und eine Rückübersetzung in Schrift. Im Falle vokaler und sprachunabhängiger Daten erfolgen sowohl Übertragung (etwa Telefon) als auch Speicherung (etwa Videokassette) maschinell bzw. elektronisch.

\subsection{Kommunikationsformen}

Betrachtet man weniger die technischen Aspekte des Medieneinsatzes und fragt statt dessen - Holly $(1996,1997)$ folgend - nach den "sozialen und kulturellen Implikationen, liegt es nahe, sie im Zusammenhang mit Kommunikationsformen zu sehen. Man kann dann fragen, was sie in Kommunikationsprozessen leisten “ (1996, S. 10). Kommunikationsformen begreift Holly (1997, S. 69) als „virtuelle Konstellationen von einem bestimmten Zeichenspeicherungs- oder Übertragungspotenzial in eine oder beide Richtungen“. Mit Blick auf solche Kommunikationsformen „sind Medien konkrete, materielle Hilfsmittel, mit denen Zeichen verstärkt, hergestellt, gespeichert und/oder übertragen werden können" (ebd., S. 69 f.). Dabei können mehrere Medien einer Kommunikationsform zugrunde liegen - etwa die Kombination von Zeichenherstellungsgeräten (etwa Stift), Materialien (etwa Tinte) und Zeichenträger (etwa

18 „Analoge Aufzeichnung - zumindest in der Form technischer Aufzeichnung - ist immer sprachlos, auch wenn in ihr sprachliche Elemente auftreten" (Hiebel et al., 1998, S. 15).

19 So wäre etwa "digitales Fernsehen“ hinsichtlich seiner technischen Erzeugung als digital, hinsichtlich seiner Darstellungsformen allerdings als analog zu begreifen.

20 Schrift gilt daher häufig auch als Medium (vgl. etwa Schmidt, 1994). Habscheid (2000, S. 136) und Holly $(1996,1997)$ plädieren dagegen dafür, Schrift als Ausdrucksform der Sprache nicht den Medien, sondern den Kommunikationsmitteln im Sinne von Zeichensystemen zuzurechnen und lediglich die Erzeugung und Speicherungsmittel von Schrift (etwa Stift, Papier, Tinte, Druck etc.) als medial zu begreifen. 
Papier) im Falle der Kommunikationsform ,Zeichnung، (vgl. hierzu Holly 1996, S. 10). Der Medieneinsatz modifiziert dabei Holly $(1996,1997)$ zufolge die Kommunikationssituation hinsichtlich der eingesetzten Zeichentypen und Kanäle, der RaumZeit-Verhältnisse, der Anzahl der (potenziellen) Teilnehmer sowie der Einrichtung des Kanals (Einweg/Mehrweg). Mithilfe dieser Dimensionen und ihrer Ausprägungen lassen sich Kommunikationsformen als Merkmalsbündel (etwa als schriftliche, öffentliche Einwegkommunikation) und konkrete verfestigte Erscheinungsformen als Typen von Medien (etwa Buch) beschreiben (vgl. dazu Holly, 1996, S. 12). Kommunikationsformen ergeben sich dann durch (Kombination von) Zeichentypen (etwa: linearstatische Schriftzeichen im Falle der Briefkommunikation) und damit zusammenhängenden Kanälen (Schrift/Brief=optischer Kanal), der Art und Weise des Umgangs mit Raum und Zeit (Brief=schriftliche Speicherung und materieller Transport), der Anzahl der Kommunikationspartner (Brief=Dyade) und ihrer Bekanntheit (Brief=privat) sowie der Möglichkeit von Wechselseitigkeit (ist im Falle der Briefkommunikation gegeben/Spezifikum: große Zeitabstände). Dabei hängen die einzelnen Faktoren im Sinne von Beschränkungen und/oder Ermöglichungen zusammen. So ist die Briefkommunikation etwa aufgrund der verwendeten Zeichentypen auf den optischen Kanal beschränkt und erzwingt aufgrund ihrer Übertragungsform (materieller Transport) eine sukzessive (im Gegensatz zu einer simultanen) zeitlich zerdehnte Form der Wechselseitigkeit.

Anhand der für Kommunikationsformen relevanten Unterscheidungen lassen sich historisch dominante Typen sozialer Austauschprozesse als Funktionskomplexe kommunikationsstrukturell bestimmen. Gegenwärtig sind das die Folgenden:

a) Medial unvermittelte, direkte oder Face-to-Face-Kommunikation (s. Schmidt in diesem Band);

b) Interpersonale, technisch vermittelte Kommunikation (etwa Telefon, Brief) ist wie Face-to-Face-Interaktionen wechselseitig und dialogisch angelegt (vgl. Höflich, 2005). Handlungen sind deshalb an jeweils konkrete Andere gerichtet. Der auf diese Weise hergestellte Kontakt ist daher zunächst nicht-öffentlich bzw. nicht an eine Öffentlichkeit gerichtet und nicht für eine solche bestimmt. Da der Kontakt mittelbar ist, ist eine Handlung der Kontaktherstellung notwendig, welche die in solchen Zusammenhängen erfolgenden Handlungen als kommunikativ bzw. als Kundgaben ausweist.

c) Massenmediale Kommunikation (etwa Presse, Rundfunk) lässt sich in Abgrenzung dazu als einseitig/nicht dialogisch, ebenfalls vermittelt, jedoch öffentlich, und als gerichtet an ein unbestimmtes Kollektiv anonymer anderer sowie im Falle des Rundfunks (Radio/Fernsehen) als fixiert, jedoch wesentlich flüchtig („Ablaufmedien") fassen. ${ }^{21}$

21 Eine grundlegende und häufig zitierte Definition findet sich bei Maletzke (1963, S. 32). Eine instruktive Erläuterung des Prozesses der Massenkommunikation sowie eine Kritik des Massenkommunikationsbegriffs findet sich bei Krotz (2007, S. $213 \mathrm{ff}$.). Siehe auch zusammenfassend und kritisch erweiternd Schmidt (2011, S. 109 ff.). 
d) Kommunikation im Rahmen sogenannter „Interaktionsmedien“ (Computer/Internet; vgl. Thiedeke, 2006) wird gefasst als eine Überlagerung einseitiger und wechselseitiger Kommunikationsformen (je nach Angebot), die für eine (Teil-)Öffentlichkeit entworfen und von einer (Teil-)Öffentlichkeit rezipiert, jedoch prinzipiell immer von einer anonymen Öffentlichkeit rezipierbar sind. ${ }^{22}$ Im Gegensatz zu den meisten im Rahmen rein interpersonalen bzw. massenmedialen Kontakts erzeugten Kommunikaten sind interaktionsmediale Kommunikate fixiert und dauerpräsent (Persistenz). Insofern lassen sie sich als Kommunikationsformen begreifen, innerhalb derer die Differenz von $b$ ) und $c$ ) innerhalb ein und desselben Mediums (Computer/Internet) entweder wiederkehrt (etwa Chat $(=b)$ vs. Homepages/ Onlineangebote $(=c)$ ) oder hybridisiert wird (etwa in Web 2.0-Angeboten, allen voran Social Media).

\section{Gesellschaftliche Leistungen}

Die Leistung der Medien besteht zunächst grundsätzlich darin, soziale Austauschprozesse unabhängiger von Raum und Zeit zu machen, indem sie Kommunikationsinhalte bzw. -prozesse fixieren (Überwindung zeitlicher Distanz) und übertragen (Überwindung räumlicher Distanz) ${ }^{23}$ : „Es (das Medium) bewahrt und vermittelt, d.h. speichert (!) und überträgt (!), Informationen über räumliche und zeitliche Distanzen hinweg und wird damit zu einem [...] Erweiterungsmittel für die menschliche Auffassungs-, Gedächtnis- und Kommunikationskompetenz" (Hiebel et al., 1998, S. 12). Während medienunvermittelte Kommunikation aufgrund ihrer Unmittelbarkeit zwar in der Lage ist, das gesamte menschliche Ausdruckvermögen in wechselseitig-simultaner Weise der Kommunikation zur Verfügung zu stellen, jedoch dadurch an das Hier und Jetzt gebunden bleibt, sind mediale Austauschformen darauf angelegt, das Hier und Jetzt durch den Einsatz von Technik zu überwinden. Die damit mehr oder weniger verbundene Auflösung von Unmittelbarkeit geht grundsätzlich zu Lasten des breiten Informationsflusses, der durch Medien reduziert wird, sowie der einfachen, simultanen Rückkopplung, die durch Medieneinsatz eingeschränkt wird. Folgt man wiederum Holly (1997, S. 65) lässt sich die Medienentwicklung „als der fortgesetzte Versuch sehen, die räumlichen und zeitlichen Beschränkungen der direkten Kommunikation zu überwinden, wobei aber jeweils wieder neue Beschränkungen entstehen“. An eine solche Vorstellung knüpft das Konzept der "Interaktivität“ an, welches erst durch das Aufkommen apparativ-technischer Medien zur massenhaft Distribution von Kommunikaten (Massenmedien) bzw. durch die Angebote und Dienstleitungen der sogenannten Interaktionsmedien virulent wurde. Wie Goertz (1995) nachvollziehbar darlegt, impliziert die Rede von „Interaktivität“ in Hinblick auf Medien nicht nur einen Vergleich mit dem Ideal einer sozialen Interaktion, sondern zudem, dass es sich um Medien handelt, deren technische Infrastruktur Interaktion zunächst

22 Daher ist auch von sogenannter „ipöK“ (interpersonal-öffentliche Kommunikation) die Rede (vgl. Marx \& Weidenacher, 2014, S. 78 f.).

23 Vgl. auch Thompson (1995, S. 31 ff.) und Schmidt \& Zurstiege (2007, S. 36 ff.). 
einschränkt ${ }^{24}$. Auf diese Weise kommt es zu einer Ausdifferenzierung von medialen Kommunikationsformen mit spezifischen Leistungspotenzialen und entsprechenden Gebrauchsmustern, die im Vergleich zu unvermittelter Kommunikation nicht als defizitär, sondern als funktional-spezifisch aufzufassen sind (vgl. Schultz, 2001) und denen im Rahmen ihrer Möglichkeit dann spezifische Problemlösefunktionen innewohnen. Auf dieser grundsätzlichen Leistung der Medien, die Ortsgebundenheit und Flüchtigkeit menschlicher Austauschprozesse zu überwinden, ruhen eine Reihe weiterer Leistungen auf, auf die abschließend stichwortartig hingewiesen werden soll:

\section{1. „Informationssysteme“}

Da menschliches Miteinander grundsätzlich an Situationen und damit an Fragen der (wechselseitigen) Wahrnehmung gebunden ist und Medien raumzeitliche Schranken zu überwinden vermögen, begreift Meyrowitz (1990 b) Medien als Teile von situationellen Informationssystemen: "[...] a social situation is [...] an ,information-system" (and) suggests that physical settings and media ,settings' are part of a continuum rather than a dichotomy" (S. 89). Meyrowitz (1990 a und b) zeigt, dass veränderte Wahrnehmungsbedingungen neue Situationen schaffen und formuliert ein Modell, wie sich Situationsdefinitionen und Verhalten durch die mediale Öffnung von Situationen verändern (vgl. ebd.; 1990 a, S. 77). Vermittelte und unvermittelte Kommunikation ist weniger als strikte Trennung als vielmehr "as part of one larger system of behavior and response“ (ebd. 1990 b, S. 67) aufzufassen. „It also brings about a complex reordering of patterns of human interaction" (Thompson, 1995, S. 82).

\subsection{Darstellung/Weltrepräsentation}

Medien leisten nicht nur einen über unmittelbare Situationen hinausgehenden Kontakt, sondern sie sind im Falle medialer (insbesondere massenmedialer) Produkte zudem kulturelle Artefakte, die etwas anderes zeigen als sich selbst. Mittels Medien kann Welt dargestellt, erzeugt und entdeckt werden (vgl. Seel, 1998). Insofern erweitern Medien die menschliche Welt enorm, da sie sowohl Vorhandenes, verdoppeln und (re-)interpretieren als auch Neues kreieren und insofern als Sinnproduzenten fungieren (vgl. Keppler, 2015). Insbesondere die Fiktionsfähigkeit medialer Darstellungen, die weit über den gesellschaftlichen Konsens hinausgehen können, ist Motor soziokulturellen Wandels.

\subsection{Verselbständigung von Kommunikaten}

Das bereits im Falle distanter Kommunikation vorhandene Auseinandertreten von Herstellung/Produktion (etwa Schreiben eines Briefs), Rezeption (Lesen eines Briefs)

24 So weist Goertz (1995) daraufhin, dass es etwa keinen Sinn macht, das Telefon als interaktiv zu bezeichnen, da dieses als interpersonales Medien per se darauf angelegt ist, interaktiven Austausch zu vermitteln. Erst im Falle der Massenmedien (Einseitigkeit des Kommunikationsflusses) bzw. der Interaktionsmedien (Kommunikation mit einer Maschine) lässt sich das Konzept der Interaktivität sinnvoll anwenden (vgl. hierzu auch Neuberger, 2007 sowie Quiring \& Schweiger, 2006). 
und dauerhaft fixiertem Produkt (Brief) schafft spätestens in seiner massenmedial ausgeprägten Form eigenständig zu untersuchende Gegenstandsbereiche der Produktion, des Produkts und der Rezeption (vgl. Willems, 2000). Pragmatisch gesehen zeichnen sich Medienprodukte durch ihre mediale Konstelliertheit aus, was sie jederzeit als auf ein Publikum bezogenen Medientext ausweist (Wulff, 2001). Insbesondere die Entstehung funktional-spezifischer Medieninstitutionen (der Berichterstattung, der Unterhaltung, der Werbung) und entsprechender genrespezifischer Medienangebote (etwa Nachrichten, fiktionaler Film oder Werbespot) rückt das mediale Produkt als Kulturware ins Zentrum des Interesses, welches einerseits kulturindustrieller Vermarktung und andererseits soziokultureller Aneignung unterliegt ${ }^{25}$. Medienangebote speisen daher zugleich eine für Identitätsbildungsprozesse eminent bedeutsame Populärkultur (im doppelten Sinne als Sphäre des Kulturwarenangebots sowie als alltäglicher Selbstinszenierungs- und Handlungsraum). Im Anschluss an Reckwitz (2006) lassen sich populärkulturelle Medien und mediale Praktiken als „Trainingsfelder der Wahrnehmung und der Kognition und der Affektivität" (ebd., S. 59) begreifen, die „vom modernen Subjekt primär als [...] Räume der Selbstformierung eingesetzt [werden]" (ebd.), was insbesondere für sozialisatorische Belange hochrelevant ist (vgl. u.a. Hoffmann \& Mikos, 2010).

\section{4. Öffentliche Kommunikation}

Erhöhte informationelle Zugänglichkeit, die Möglichkeit der Repräsentation von Welt sowie die Eigenständigkeit medialer Angebote schafft einen Bereich öffentlicher Kommunikation, welcher besonderen Bedingungen und Strukturmerkmalen unterliegt und als eigenständiges Mediensystem mit spezifischer Funktionalität beschreibbar ist. Insbesondere die sogenannten Massenmedien schaffen durch ihre besondere Form der Kommunikation, die in der Lage ist, potenziell alle Gesellschaftsmitglieder zu erreichen, Öffentlichkeit, verstanden als ein für jedermann zugänglicher „Kommunikationsraum", der - spätestens seit Jürgen Habermas (1974) - über seine gesellschaftliche Funktion definiert ist ${ }^{26}$. Diese besteht darin, die Versorgung der Gesellschaftsmitglieder mit relevanten Informationen sicherzustellen, um so einen öffentlichen Diskurs als Grundlage demokratischer Willensbildung zu gewährleisten (Ideal einer deliberativen Öffentlichkeit). Zugleich konstruieren Medien in und durch ihre Darstellungen Realität(en) und liefern so Selbstbeschreibungen der Gesellschaft (vgl. Luhmann, 1997, 1996). Voraussetzung hierfür ist, dass Prozesse der Massenkommu-

25 An dem Warencharakter des Medienangebots setzt die Kritik einer Kulturindustrie an (vgl. grundsätzlich Horkheimer \& Adorno, 1997; zusammenfassend Göttlich, 2003). Zum Konzept der Aneignung vgl. grundsätzlich Faber, 2001; Holly \& Püschel, 1993; Winter, 1995. Insbesondere die sogenannten Cultural Studies (vgl. Hepp, 1999) betonen diesen Doppelcharakter populärkultureller (Medien-)Produkte (vgl. grundlegend Fiske, 1989, S. 23 ff., zusammenfassend Hepp, 1999, S. 66 ff.).

26 Erst auf der Grundlage von technischen Kommunikationsmitteln (Medien) - so Hickethier (2010, S. 208 ff.) - wurde es möglich, „den Ort, an dem über öffentliche Angelegenheiten kommuniziert wurde, von den Beteiligten zu trennen". So wurzelt der Begriff der Öffentlichkeit zwar in Raumkonzepten (Forum, Arena, Salon), fungiert allerdings im Rahmen von massenmedialen Öffentlichkeitskonzepten lediglich noch als Metapher. 
nikation ,interaktionsfrei [...] operieren" (Sutter, 2001, S. 25), da erst auf diese Weise eine erhöhte Verbreitung und Definitionsmacht von Kommunikaten möglich ist (vgl. Wehner, 1997 sowie Schneider, 2001)27.

\subsection{Kulturelle Folgen}

Da Medien nicht nur Zeichen übermitteln, sondern Kommunikationsformen in wesentlicher Hinsicht prägen, können sie auch als „Dispositive“ im Sinne Foucaults (1977) und Baudrys (1977) begriffen werden. Medien sind in dieser Hinsicht wirklichkeitskonstituierende „Apparate“ (vgl. Krämer, 1998), die nicht bloß übertragen, sondern im und durch diesen Prozess Wahrnehmungs-, Denk und Kommunikationsweisen wesentlich prägen ${ }^{28}$. Auf dieser Vorstellung gründet die viel zitierte Formel Marshall McLuhans (1970, S. 17), "the medium is the message“, welche die der Botschaft geltende Aufmerksamkeit auf das Medium selbst lenkt ${ }^{29}$. Mediendispositive bezeichnen gesellschaftlich institutionalisierte Wahrnehmungs- und Kommunikationsarrangements und zeichnen sich dadurch aus, dass sie implizit regeln, wie etwas hervorgebracht und wahrgenommen wird. ${ }^{30}$ Medien in diesem Sinn zeigen nicht bloß etwas oder verbreiten Inhalte und Informationen, sondern definieren wie Welt erzeugt und wahrgenommen wird und prägen so Gesellschaften nachhaltig.

\section{Fazit}

Ein mediensoziologischer Begriff von Medien setzt an Techniken der Vermittlung von Kommunikations- und Interaktionsprozessen an und reflektiert insbesondere die durch solche Vermittlungsprozesse in Gang gesetzten Veränderungen sozialen Austauschs. Medienvermittlung verändert die Grundbedingungen sozialen Austauschs, indem nicht gegebene Anwesenheit überbrückt und dadurch wechselseitiger Zugang transformiert wird. Zudem modifizieren die dazu eingesetzten Techniken den Symbolgebrauch (Kanalkapazität) sowie das Kommunikationsverhältnis, was zugleich neue Kommunikations- und Verbreitungsformen (etwa auf Speicher-, Reproduktions-

27 Der in diesem Zusammenhang entscheidende Terminus des „Massenpublikums lässt sich [...] als zu unterstellende zeitgleiche informationelle Synchronisierung sämtlicher Mitglieder einer Gesellschaft deuten“ (Wehner, 1997, S. 101). Insofern „erzeugen Massenmedien eine Art ,Kollektivbewusstsein“" (ebd.).

28 Tholen (2005) weist in diesem Zusammenhang mit Blick auf Wahrnehmung und Welterkenntnis etwa auf den „unauflöslichen Zusammenhang von Aisthesis (Wahrnehmung) und Medialität...“ (ebd., S. 162) hin. Böhme-Dürr (2003) betont unter Rekurs auf Posner (1985) „die wechselseitige, unentrinnbare Abhängigkeit von Semioseart und Medienbeschaffenheit " (ebd., S. 358).

29 Vgl. zusammenfassend Kloock \& Spahr (2000, S. 39 ff.). Einen anschaulichen Vergleich liefert Meyrowitz (1987, S. 56): „wenn wir uns in der öffentlichen wie wissenschaftlichen Aufmerksamkeit vor allem auf den Inbalt von Kommunikationen konzentrieren, gleicht das dem hypothetischen Versuch, die Bedeutung des Automobils zu verstehen, indem man ignoriert, dass es ein neues Transportmittel gibt, und sich stattdessen auf eine detaillierte Untersuchung der Namen und Gesichter von Passagieren konzentriert".

30 Vgl. Hickethier (2010, S. 186 ff.) sowie Paech (2003). 
und Übertragungsmedien beruhende Massenkommunikation) ermöglicht. Insgesamt verändern Medien auf diese Weise die Bedingungen und Möglichkeiten einer gesellschaftlichen Konstruktion von Wirklichkeit (Berger \& Luckmann, 1969) nachhaltig.

Interaktion und Kommunikation als Grundlage gesellschaftlicher Wirklichkeitskonstruktion und deren mediale Realisierung stehen dabei in einem Verhältnis zunehmender Sinnkomplexität: Nicht-zentrierte Interaktion oder Anwesenheit (=einfaches Sozialsystem) macht Verhalten kommunikativ und Kommunikation semiotisiert Wahrnehmung (s. Schmidt in diesem Band). Der Gebrauch von Medien tut beides: er macht Verhalten und Wahrnehmung kommunikativ. Dies ist insofern für mediensoziologische Belange besonders bedeutsam, da das (massen- und dauerhafte) Abheben sozialer Austauschprozesse vom Hier und Jetzt für Gesellschaftsdiagnosen hoch relevant ist.

\section{Leseempfehlungen}

Böhme-Dürr, Karin (2003). Technische Medien der Semiose. In R. Posner, K. Robering \& T. A. Sebeok (Hrsg.), Semiotik/Semiotics. Ein Handbuch zu den zeichentheoretischen Grundlagen von Natur und Kultur. Berlin/New York: de Gruyter. S. 357-384

Hickethier, Knut (2010). Einführung in die Medienwissenschaft. Stuttgart: Metzler.

Hiebel, Hans H., Hiebler, Heinz, Kogler, Karl \& Walitsch, Herwig (1998). Die Medien: Logik - Leistung - Geschichte. München: Fink.

Jäckel, Michael (Hrsg.). (2005). Mediensoziologie: Grundfragen und Forschungsfelder. Wiesbaden: VS.

Thompson, John B. (1995). The media and modernity: A social theory of the media. Cambridge: Polity Press.

\section{Literatur}

Auslander, Philip (2008). Liveness: Performance in a mediatized culture. London: Routledge.

Baudry, Jean-Louis (1977). Das Dispositiv: Metapsychologische Betrachtungen des Realitätseindrucks. Psyche, 48, (11), 1047-1074.

Berger, Peter L. \& Luckmann, Thomas (1969). Die gesellschaftliche Konstruktion der Wirklichkeit: Eine Theorie der Wissenssoziologie. Frankfurt a. M.: Fischer.

Böhme-Dürr, Karin (2003). Technische Medien der Semiose. In R. Posner, K. Robering \& T. A. Sebeok (Hrsg.), Semiotik/Semiotics: Ein Handbuch zu den zeichentheoretischen Grundlagen von Natur und Kultur. Berlin/New York: de Gruyter. S. 357-384.

Faber, Marlene (2001). Medienrezeption als Aneignung. In W. Holly, U. Püschel \& J. Bergmann (Hrsg.), Der sprechende Zuschauer - wie wir uns Fernsehen kommunikativ aneignen. Wiesbaden: Westdeutscher Verlag. S. 25-40.

Faulstich, Werner (2004). Medienwissenschaft. München: Fink.

Fiske, John (1989). Understanding popular culture. London: Taylor \& Francis.

Foucault, Michel (1977). Der Wille zum Wissen: Sexualität und Wabrheit (Bd. 1). Frankfurt a. M.: Suhrkamp.

Goertz, Lutz (1995). Wie interaktiv sind Medien: Auf dem Weg zu einer Definition von Interaktivität. Rundfunk und Fernsehen, 43, (4), 477-493.

Göttlich, Udo (2003). Kulturindustrie. In H. Hügel (Hrsg.), Handbuch Populäre Kultur. Stuttgart: Metzler. S. 45-48. 
Graumann, Carl Friedrich (1972). Interaktion und Kommunikation. In C.F. Graumann (Hrsg.), Handbuch der Psychologie: Sozialpsychologie (Bd. 7). Göttingen, S. 1109-1262.

Gumbrecht, Hans Ulrich \& Pfeiffer, Karl Ludwig (Hrsg.). (1988). Materialität der Kommunikation. Frankfurt a. M.: Suhrkamp.

Habermas, Jürgen (1974). Strukturwandel der Öffentlichkeit. Neuwied: Luchterhand.

Habscheid, Stephan (2000). 'Medium' in der Pragmatik: Eine kritische Bestandsaufnahme. Deutsche Sprache, 28, (2), 126-143.

Hepp, Andreas (1999). Cultural Studies und Medienanalyse: Eine Einfübrung. Opladen: Westdeutscher Verlag.

Hickethier, Knut (1995). Dispositiv Fernsehen: Skizze eines Modells. Montage AV, 4, (1), 63-84.

Hickethier, Knut (2010). Einführung in die Medienwissenschaft. Stuttgart: Metzler.

Hiebel, Hans H., Hiebler, Heinz,Kogler, Karl \& Walitsch, Herwig (1998). Die Medien: Logik - Leistung Geschichte. München: Fink.

Höflich, Joachim R. (2005). Medien und interpersonale Kommunikation. In M. Jäckel (Hrsg.), Mediensoziologie: Grundfragen und Forschungsfelder. Wiesbaden: Springer. S. 69-90.

Hoffmann, Dagmar \& Mikos, Lothar (Hrsg.). (2010). Mediensozialisationstheorien: Modelle und Ansätze in der Diskussion (2. Aufl.). Wiesbaden: VS.

Holly, Werner (1996). Zur inneren Logik der Mediengeschichte. In B. Rüschoff \& U. Schmitz (Hrsg.), Kommunikation und Lernen mit alten und neuen Medien. Frankfurt a. M.: Lang. S. 9-16.

Holly, Werner (1997). Zur Rolle von Sprache in Medien: Semiotische und kommunikationsstrukturelle Grundlagen. Muttersprache, 107, (1), 64-75.

Holly, Werner \& Püschel, Ulrich (Hrsg.). (1993). Medienrezeption als Aneignung: Methoden und Perspektiven qualitativer Medienforschung. Opladen: Westdeutscher Verlag.

Horkheimer, Max \& Adorno, Theodor W. (1997). Kulturindustrie: Aufklärung als Massenbetrug. In G. Schmid-Noerr (Hrsg.), Max Horkheimer - Gesammelte Schriften: 'Dialektik der Aufklärung' und Schriften 1940-1950 (Bd. 5). Frankfurt a. M.: Fischer. S. 144-196.

Jäckel, Michael (2005 a). Mediensoziologie: Grundfragen und Forschungsfelder. Wiesbaden: VS.

Jäckel, Michael (2005 b). Medienwirkungen: Ein Studienbuch zur Einführung. Wiesbaden: VS.

Keppler, Angela (2015). Das Fernsehen als Sinnproduzent: Soziologische Fallstudien. Berlin et al.: de Gruyter Oldenbourg.

Kloock, Daniela \& Spahr, Angela (2000). Medientheorien: Eine Einfübrung. München: Fink.

Krämer, Sybille (1998). Das Medium als Spur und als Apparat. In S. Krämer (Hrsg.), Medien - Computer - Realität: Wirklichkeitsvorstellungen und neue Medien. Frankfurt a. M.: Suhrkamp. S. 73-94.

Krotz, Friedrich (2007). Mediatisierung: Fallstudien zum Wandel von Kommunikation. Wiesbaden: VS.

Kübler, Hans-Dieter (2000). Mediale Kommunikation. Tübingen: Niemeyer.

Leschke, Rainer (2007). Einführung in die Medientheorie. München: Fink.

Luhmann, Niklas (1996). Die Realität der Massenmedien. Opladen: Westdeutscher Verlag.

Luhmann, Niklas (1997). Die Gesellschaft der Gesellschaft (Bd. 1-2). Frankfurt a. M.: Suhrkamp.

Maletzke, Gerhard (1963). Psychologie der Massenkommunikation: Theorie und Systematik. Hamburg: Hans-Bredow-Institut.

Marx, Konstanze \& Weidacher, Georg (2014). Internetlinguistik: ein Lehr- und Arbeitsbuch. Tübingen: Narr.

McLuhan, Marshall (1970). Die magischen Kanäle: Understanding Media. Frankfurt a. M.: Fischer.

Meyrowitz, Joshua (1987). Die Fernseh-Gesellschaft: Wirklichkeit und Identität im Medienzeitalter. Weinheim: Beltz.

Meyrowitz, Joshua (1990 a). Using Contextual Analysis to Bridge the Study of Mediated and Unmediated Behavior. In B. D. Ruben \& L. A. Lievrouw (Hrsg.), Mediation, Information and Communication: Information and Behavior (Bd. 3). New Brunswick: Transaction Press. S. 67-94.

Meyrowitz, Joshua (1990 b). Redefining the Situation: Extending Dramaturgy into a theory of social change and media effects. In S. H. Riggins (Hrsg.), Beyond Goffman: studies on communication, institution, and social interaction. Berlin: de Gruyter. S. 65-97. 
Mock, Thomas (2006). Was ist ein Medium? Eine Unterscheidung kommunikations- und medienwissenschaftlicher Grundverständnisse eines zentralen Begriffs. Publizistik, 51, (2), 183-200.

Münker, Stefan \& Roesler, Alexander (Hrsg.). (2008). Was ist ein Medium? Frankfurt a. M.: Suhrkamp.

Neuberger, C. (2007). Interaktivität, Interaktion, Internet. Publizistik, 52, (1), 33-50.

Paech, Joachim (2003). Überlegungen zum Dispositiv als Theorie medialer Topik. In F. Albersmeier (Hrsg.), Texte zur Theorie des Films. Stuttgart: Reclam. S. 465-498.

Posner, Roland (1985). Nonverbale Zeichen in öffentlicher Kommunikation: Zu Geschichte und Gebrauch der Begriffe 'verbal' und 'nonverbal', 'Interaktion' und 'Kommunikation', 'Publikum' und 'Öffentlichkeit', 'Medium' und 'Massemedium' und 'multimedial'. Zeitschrift für Semiotik, 7, (3), 235-271.

Pross, Harry (1972). Medienforschung: Film, Funk, Presse, Fernsehen. Darmstadt: Habel.

Quiring, Oliver \& Schweiger, Wolfgang (2006). Interaktivität - ten years later: Bestandsaufnahmen und Analyserahmen. Medien ơ Kommunikationswissenschaft, 54, (1), 5-24.

Reckwitz, Andreas (2006). Das hybride Subjekt: eine Theorie der Subjektkulturen von der bürgerlichen Moderne zur Postmoderne. Weilerswist: Velbrück.

Sandbothe, Mike (2003). Medien - Kommunikation - Kultur: Grundlagen einer pragmatischen Kulturwissenschaft. In F. Jaeger, B. Liebsch, J. Rüsen \& J. Straub (Hrsg.), Handbuch der Kulturwissenschaften: Grundlagen und Schlüsselbegriffe (Bd. 1). Stuttgart/Weimar: Metzler. S. 119-127.

Saussure, Ferdinand de (1967). Grundfragen der allgemeinen Sprachwissenschaft. Berlin/New York: de Gruyter.

Schmidt, Axel (2011). Medien I Interaktion: Zum Zusammenhang von Handeln und Darstellen am Beispiel faktualer Fernsehformate. Baden-Baden: Nomos.

Schmidt, Siegfried J. (1994). Kognitive Autonomie und soziale Orientierung: Konstruktivistische Bemerkungen zum Zusammenhang von Kognition, Kommunikation, Medien und Kultur. Frankfurt a. M.: Suhrkamp.

Schmidt, Siegfried J. \& Zurstiege, Guido (2007). Kommunikationswissenschaft: Systematik und Ziele. Reinbek bei Hamburg: Rowohlt.

Schneider, Wolfgang Ludwig (2001). Intersubjektivitätsproduktion in Interaktion und Massenkommunikation. In T. Sutter \& M. Charlton (Hrsg.), Massenkommunikation, Interaktion und soziales Handeln. Wiesbaden: Westdeutscher Verlag. S. 84-110.

Schneider, Wolfgang Ludwig (2002). Grundlagen der soziologischen Theorie: Garfinkel-RC-Habermas - Lubmann (Bd. 2). Wiesbaden: Westdeutscher Verlag.

Schütz, Alfred \& Luckmann, Thomas (1979). Strukturen der Lebenswelt (Bd. 1). Frankfurt a. M.: Suhrkamp.

Schultz, Tanjev (2001). Mediatisierte Verständigung. Zeitschrift für Soziologie, 30, (2), 85-102.

Schweinitz, Jörg (1994). 'Genre' und lebendiges Genrebewusstsein. Montage AV, 3, (2), 99-118.

Seel, Martin (1998). Medien der Realität und Realität der Medien. In S. Krämer (Hrsg.), Medien - Computer-Realität: Wirklichkeitsvorstellungen und neue Medien. Frankfurt a. M.: Suhrkamp. S. 244-268.

Sutter, Tilmann (2001). Sinnstrukturen der Medienkommunikation. In T. Sutter \& M. Charlton (Hrsg.), Massenkommunikation, Interaktion und soziales Handeln. Wiesbaden: Westdeutscher Verlag. S. 21-45.

Thiedeke, Udo (2006). Interaktionsmedien. In L. R. Tsvasman (Hrsg.), Das große Lexikon Medien und Kommunikation: Kompendium interdisziplinärer Konzepte. Würzburg: Ergon. S. 160-163.

Tholen, Georg Christoph (2005). Medium/Medien. In A. Roesler \& B. Stiegler (Hrsg.), Grundbegriffe der Medientheorie. Paderborn: Fink. S. 150-172.

Thompson, John B. (1995). The media and modernity: A social theory of the media. Cambridge: Polity Press.

Wehner, Josef (1997). Interaktive Medien - Ende der Massenkommunikation? Zeitschrift für Soziologie, 26, (2), 96-114.

Willems, Herbert (2000). Medienproduktion, Medienprodukt, Medienrezeption. Medien \& Kommunikationswissenschaft, 48, (2), 212-225.

Winter, Rainer (1995). Der produktive Zuschauer: Medienaneignung als kultureller und ästhetischer Prozess. München: Quintessenz. 
Wulff, Hans J. (1978). Medium und Kanal: Zur Terminologie der Semiotik (Bd. 1). Münster: MAKs. S. 37-67.

Wulff, Hans J. (2001). Konstellationen, Kontrakte und Vertrauen: Pragmatische Grundlagen der Dramaturgie. Montage AV, 10, (2), 131-154.

Ziemann, Andreas (2006). Soziologie der Medien. Bielefeld: transcript. 